\title{
Interaction Techniques for HMD-HHD Hybrid AR Systems
}

\author{
Rahul Budhiraja* Gun A. Lee ${ }^{\dagger} \quad$ Mark Billinghurst ${ }^{\ddagger}$ \\ The Human Interface Technology Laboratory New Zealand, University of Canterbury
}

\begin{abstract}
Most mobile Augmented Reality (AR) systems use either a head mounted display (HMD) or a handheld display (HHD) as a hardware platform. As mobile devices become more affordable, it becomes more common that users own more than one mobile device and use them together. In this research, we investigate Hybrid AR systems that use both HMD and HHD for AR visualization and interaction. In addition to a simple approach of using HMD as a display and HHD as an input device (e.g. a touch pad or a pointer), we further explore novel interaction techniques that can take advantage of having both HMD and HHD closely integrated into one AR system, such as cross-device information sharing, situation adaptive visualization management, and multilayered visualization.
\end{abstract}

Keywords: Mobile augmented reality, wearable computer, headmounted display, handheld display.

Index Terms: H.5.1 [Information Interfaces and Presentation]: Multimedia Information Systems - Artificial, augmented, and virtual realities; H.5.2 [Information Interfaces and Presentation]: User Interfaces - Interaction styles

\section{INTRODUCTION}

Most mobile Augmented Reality (AR) systems use either a head mounted display (HMD) or a handheld display (HHD) as a hardware platform. After smartphones got popular, HHDs became one of the representative hardware platforms for AR applications. Taking benefit from a touch screen and various other sensors, HHDs are intuitive to interact with, compared to HMDs that has advantages more on immersive visualization. While not as widely adopted as HHDs, HMDs have their own merit as an AR display such as being always worn in front of the user's view.

As mobile devices become more affordable, it is expected to be more common for users to have more than one mobile device and use them together. In this research, we investigate Hybrid AR systems that use both HMD and HHD for AR visualization and interaction. While there has been research on interaction methods for AR systems using either HMD or HHD, less work has been done on systems combining both HMD and HHD. We think this is particularly important as new lightweight HMDs are becoming more affordable (e.g. Google Glass), while HHDs are already widely adopted.

\section{HMD-HHD HYBRID AR SYSTEM}

A simple approach to use HHD with HMD in an AR system

\footnotetext{
* e-mail: rahul.budhiraja@canterbury.ac.nz

† e-mail: gun.lee@canterbury.ac.nz

† e-mail: mark.billinghurst@canterbury.ac.nz
}

IEEE International Symposium on Mixed and Augmented Reality 2013 Science and Technology Proceedings

1 - 4 October 2013, Adelaide, SA, Australia

978-1-4799-2869-9/13/\$31.00 @2013 IEEE would be to use HHD as a secondary display or as an input device. The Touring Machine [2], one of the earliest mobile AR systems, made use of a HHD as a secondary display in combination with a HMD. The system used a tablet computer with a stylus input for showing additional information about the virtual content shown on the HMD. Henderson et al. [5] used a smartphone attached on user's wrist as a secondary display showing graphical user interfaces to control a presentation being viewed on a HMD, while Ha [4] used HHD as a pure input device, which is a similar case with many wearable computing systems that use various kinds of handheld input devices together with HMD [7].

With touch interfaces becoming widely used on HHDs, we have are looking into using the touch screen or motion sensor on the HHD to control a cursor on the HMD view. And in addition to this simple approach, we are investigating interaction techniques that can take advantage of having both HMD and HHD closely integrated into one AR system.

\subsection{Cross-device Swipe Gesture}

For showing additional information on the HHD about the objects in the HMD view, we propose cross-device swipe gesture as an intuitive interaction technique. Swiping is similar to dragging an object from one display onto another and feels natural on a monocular HMD and HHD. Using this method, the user can swipe horizontally on the HHD to show additional information about the object selected on the HMD screen. For example if the HMD is worn covering the right eye and the HHD is held in the left hand, a swiping gesture on the HHD from right to left could be applied. Figure 1 shows an example of performing the cross-device swipe gesture on the HHD (the left side of the figure) to get additional information about the object on the HMD view (the right side of the figure)

The method could also be applied to other tasks, such as moving objects from the HHD screen into the AR HMD view. For example, the HHD could show a list of objects as 2D icons and when one is selected and then swiped off screen, it could appear in the AR view as a 3D object. This would enable a user to quickly place objects in the AR scene.

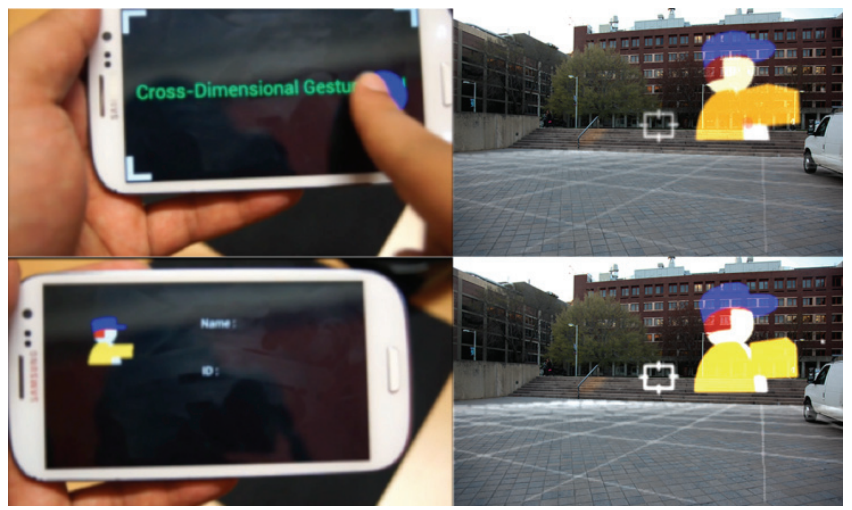

Figure 1: Cross-device swipe gesture 


\subsection{Multi-layered Visualization}

In addition to showing 2D information on HHD, it can be also used to show AR visualization together with the HMD. Compared to previous work that focused on providing a secondary higher resolution display [1][3], the proposed style of visualization enables the user to have different representation of the same 3D scene in the AR view using the HHD as another layer of visualization.

In our scenario, the user could hold the HHD in front of their face which is visible through the HMD, and the handheld display shows another style of visualization (e.g. X-ray vision) of the current AR scene. This is useful in situations where you want to look at different versions of the virtual objects in the AR scene. For example, if you want to look at the interior of a building or an older version of an augmented building. The HHD thus works like a portable magic lens [6] that when brought in front of the users view "augments the augmented" scene.

To achieve this, the HHD can be tracked from the camera mounted on the HMD, and the region on the HMD's view covered by the HHD can be rendered transparent (or black for optical seethrough HMDs) to let the user see the content shown on the HHD screen. Figure 2 shows a view from a prototype system which we built for demonstrating the multi-layered visualization. The colored papers attached on the corners of the HHD is used for tracking the HHD, while the large display on the back is showing the view on the HMD (for testing purpose).

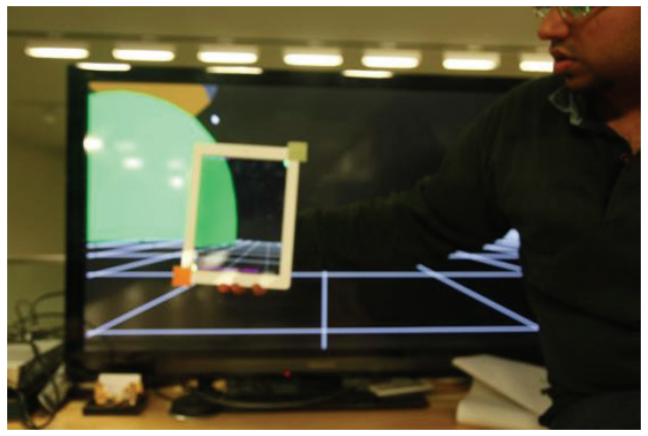

Figure 2: Multi-layered visualization

\subsection{Situation Adaptive Visualization Management}

When using both HMD and HHD as displays, the view can be cluttered with too much information. To avoid this, we are investigating situation adaptive visualization where the information shown on the HMD and HHD depends on the relationship between the devices and device orientation. The overall goal is to provide different viewing functionalities in a single application while preserving the fluidity of the experience, and not requiring the user to explicitly change viewing modes.

To achieve this, we track the motion of both HMD and HHD to recognize their relative pose. Figure 3 shows how the HMD and HHD can show different views based on the situation. (A) When the user looks down at the HHD, the HMD turns transparent to help user have clearer view of the HHD. One example use of this could be switching from the AR view shown on the HMD to a 2D map shown on the HHD. (B) When the user looks away from the HHD, the HMD view shows the AR View and the smartphone can be used as a gestural input device. (C) When the user puts the HHD in the upright position, the HHD can be used for showing additional details of the virtual object on the HMD or can be used as for multi-layered visualization as explained in the previous section.
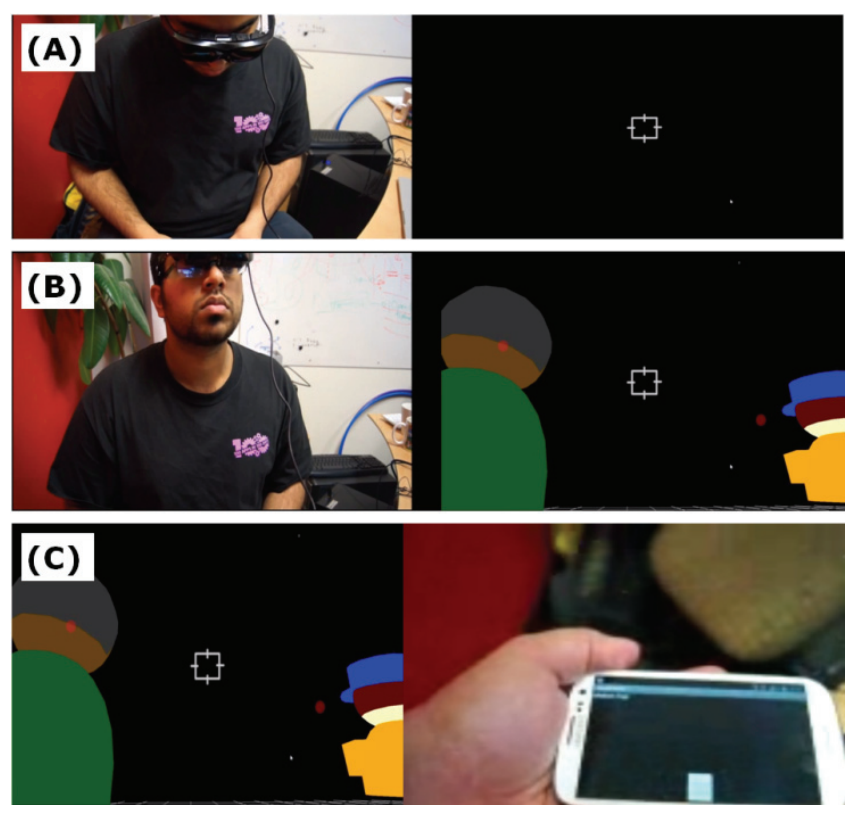

Figure 3: Situation adaptive view management

\section{CONCLUSION}

In this research, we are investigating visualization and interaction methods for hybrid AR systems that use both HMD and HHD. In addition to using HMD as a display and HHD as an input device, we especially focus on exploring novel interaction and visualization techniques that can take advantage of having both HMD and HHD closely integrated into one AR system, such as cross-device swiping gesture, multi-layered visualization, and situation adaptive visualization management. We are planning to investigate the usefulness of the proposed interaction techniques through user studies, and to explore the application space that this kind of hybrid AR systems could be ideal for.

\section{REFERENCES}

[1] H. Benko, E. W. Ishak, and S. Feiner. Collaborative mixed reality visualization of an archaeological excavation. In Proc. of the $3 r d$ IEEE and ACM Intl. Symp. on Mixed and Augmented Reality (ISMAR 2004), pp. 132-140, 2004.

[2] S. Feiner, B. MacIntyre, T. Höllerer, and T. Webster. A touring machine: Prototyping 3D mobile augmented reality systems for exploring the urban environment. In Proc. of the 1st IEEE Intl. Symp. on Wearable Computers(ISWC '97), pp. 74-81, 1997.

[3] S. Feiner and A. Shamash. Hybrid user interfaces: breeding virtually bigger interfaces for physically smaller computers. In Proc. of the 4th ACM Symp. on User Interface Software and Technology (UIST'91), pp. 9-17, 1991.

[4] T. Ha and W. Woo. ARWand: Phone-based 3d object manipulation in augmented reality environment. In Proc. of Intl. Symp. on Ubiquitous Virtual Reality (ISUVR '11), pp. 44-47, 2011.

[5] S. J. Henderson and S. Feiner. Evaluating the benefits of augmented reality for task localization in maintenance of an armored personnel carrier turret. In Proc. of the 8th IEEE Intl. Symp. on Mixed and Augmented Reality (ISMAR '09), pp. 135-144, 2009.

[6] J. Looser, M. Billinghurst, and A. Cockburn. Through the looking glass: the use of lenses as an interface tool for Augmented Reality interfaces. In Proc. of the 2nd Intl. Conf. on Computer graphics and interactive techniques in Australasia and South East Asia (GRAPHITE '04), pp. 204-211, 2004.

[7] B. Thomas, S. Tyerman, and K. Grimmer. Evaluation of three input mechanisms for wearable computers. In Proc. of Intl. Symp. on Wearable Computers (ISWC '97), pp. 2-9, 1997. 